Document downloaded from:

http://hdl.handle.net/10251/121429

This paper must be cited as:

Burgos-Simon, C.; Cortés, J.; Villafuerte, L.; Villanueva Micó, RJ. (2018). Solving random mean square fractional linear differential equations by generalized power series: analysis and computing. Journal of Computational and Applied Mathematics. 339:94-110. https://doi.org/10.1016/j.cam.2017.12.042

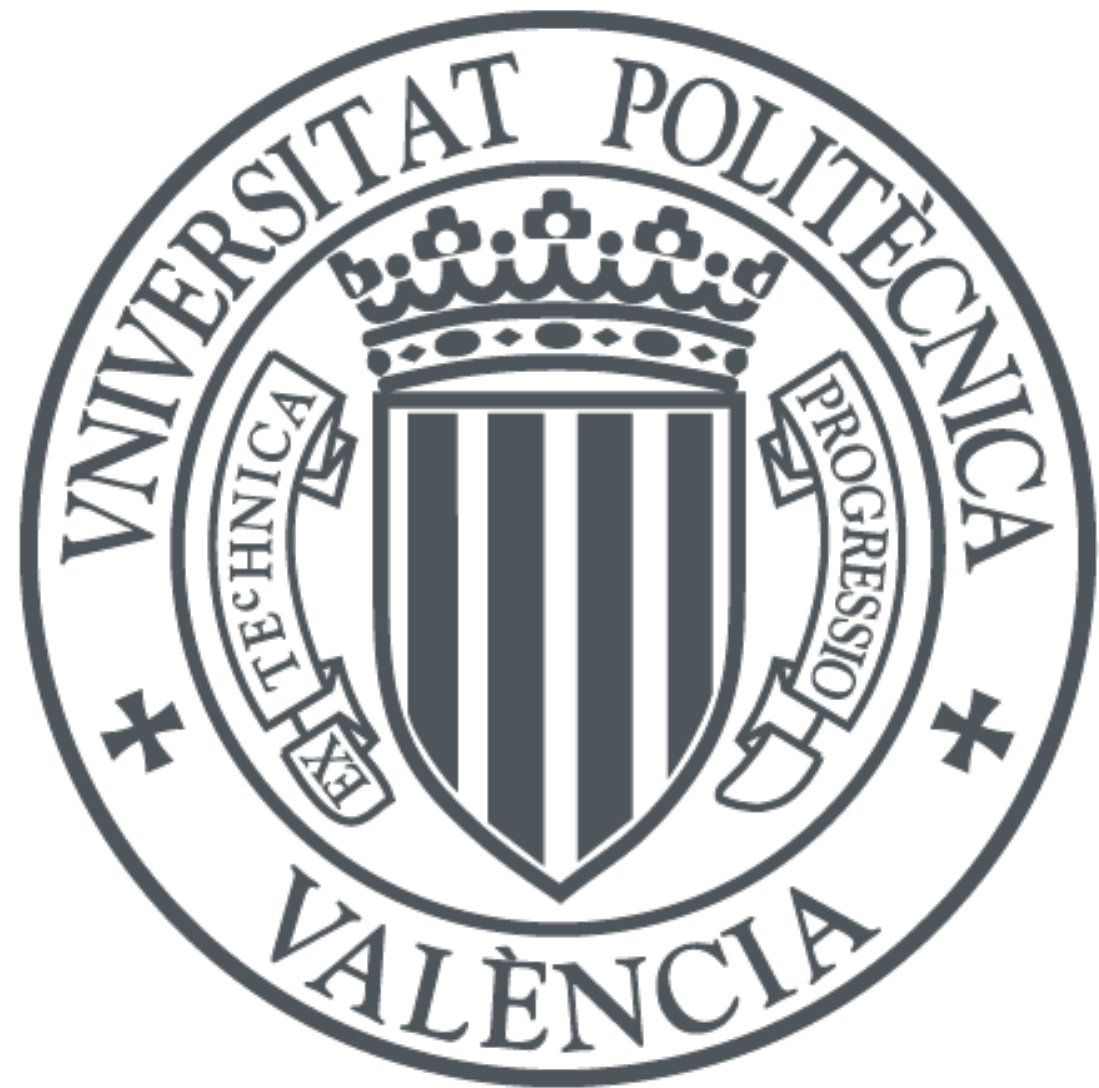

The final publication is available at

http://doi.org/10.1016/j.cam.2017.12.042

Copyright Elsevier

Additional Information 


\title{
Solving random mean square fractional linear differential equations by generalized power series: analysis and computing
}

\author{
C. Burgos ${ }^{\mathrm{a}}$, J.-C. Cortés ${ }^{\mathrm{a}}$, L. Villafuerte ${ }^{\mathrm{b}}$, R.J. Villanueva ${ }^{\mathrm{a}}$ \\ ${ }^{a}$ Instituto Universitario de Matemática Multidisciplinar, \\ Universitat Politècnica de València, \\ Camino de Vera s/n, 46022, Valencia, Spain \\ ${ }^{b}$ Department of Mathematics, \\ University of Texas at Austin
}

\begin{abstract}
This paper deals with solving the general random (Caputo) fractional linear differential equation under general assumptions on random input data (initial condition, forcing term and diffusion coefficient). Our findings extend, in two directions, the results presented in a recent contribution by the authors. In that paper, a mean square random generalized power series solution has been constructed in the case that the fractional order, say $\alpha$, of the Caputo derivative lies on the interval $] 0,1]$ and assuming that the diffusion coefficient belongs to a class, $\mathfrak{C}$, of random variables that contains all bounded random variables. However, significant families of unbounded random variables, such as Gaussian and Exponential, for example, do not fall into class $\mathfrak{C}$. Now, in this contribution we first enlarge the class of random variables to which the diffusion coefficient belongs and we prove that the constructed random generalized power series solution is mean square convergent too. We show that any bounded random variable and important unbounded random variables, including Gaussian and Exponential ones, are allowed to play the role of the diffusion coefficient as well. Secondly, we construct a mean square random generalized power series solution in the case that $\alpha$ parameter lies on the larger interval ]0,2]. As a consequence, the results established in our previous contribution are fairly generalized. It is particularly enlightening, the numerical study of the convergence of the approximations to the mean and the standard deviation of the solution stochastic process in terms of $\alpha$ parameter and on the type of the probability distribution chosen for the diffusion coefficient.
\end{abstract}

Keywords: random linear fractional differential equation, random mean square convergence, random mean square Caputo fractional derivative

\section{Introduccion and motivation}

The ubiquity of differential equations for modelling successfully real problems in different realms as Physics, Economics, Epidemiology, etc., is well-known. When they are applied to

\footnotetext{
*Corresponding author

Email addresses: clabursi@posgrado.upv.es (C. Burgos), jccortes@imm.upv.es (J.-C. Cortés), lva5@hotmail.com (L. Villafuerte), rjvillan@imm.upv.es (R.J. Villanueva)
} 
4 describe the dynamics of physical phenomena on the basis of sampled data, the parameters of 5 differential equations (coefficients, forcing term, initial/boundary conditions) need to be fixed. This is usually done by assigning a nominal or averaged value (estimate), thus deterministic, to each model parameter. Although this is often accepted, in the context of modelling it is more natural to interpret parameters of differential equations as random variables or stochastic processes rather than constants and functions, respectively. This is fairly justified because involved measurement errors and inherent complexity usually encountered when modelling real phenomena. This simple but realistic arguments justify the study of differential equations considering uncertainty in their formulation. Two classes of such differential equations are often distinguised, namely, Stochastic Differential Equations (SDEs) and Random Differential Equations (RDEs). In dealing with SDEs, the uncertainty is forced by a stochastic process whose sample behaviour is quite irregular, such as the Wiener process whose trajectories are nonwhere differentiable. In this case, the underlying probabilistic pattern is Gaussian. Solving exact or numerically these type of equations requires the application of Itô Stochastic Calculus [1, 2]. RDEs appear as natural generalizations of their deterministic counterpart, namely determinisitic differential equations, since they are just formulated by randomizing their parameters. This is fairly advantageous on both theoretical and practical levels. From a theoretical point of view, solving RDEs is based on Mean Square Random Calculus whose operational rules take advantage of powerful classical Newton-Leibniz Calculus. Indeed, in this context the probabilistic concepts of mean square continuity, differentiability, integrability of a stochastic process can be characterized in terms of classical continuity, differentiability, integrability to its associated correlation function, which is a two-dimensional deterministic function $[3,4,5]$. From a practical standpoint, a wide range of probabilistic distributions are allowed for input parameters including the Gaussian pattern, although assuming on them regular behaviour (like sample continuity) [6,7]. Apart from considering uncertainty in differential equations, mathematical modelling can be improved when fractional derivatives are also introduced. This can be clearly justified because fractional derivatives are parametrizations, via the order of the fractional derivative, of powerful concept of classical derivative. Naturally, this allows more flexibility when fitting the solution of a random fractional differential equation to sample data $[8,9,10]$. This leads to the emergent and attractive realm of fractional SDEs and RDEs, where two powerful tools, namely Fractional Calculus and Itô Stochastic/Mean Square Random Calculus, are combined. Some recent contributions dealing with interesting problems related to fractional SDEs and fractional RDEs include [11, 12] and $[13,14,15,16,17,18]$, respectively.

In this paper we deal with the following random fractional initial value problem (IVP)

$$
\left\{\begin{array}{cl}
\left({ }^{C} D_{0^{+}}^{\alpha} Y\right)(t)-\lambda Y(t) & =\gamma, \quad t>0, \quad 0<\alpha \leq 2, \\
Y^{(j)}(0) & =\beta_{j}, \quad 0 \leq j \leq-[-\alpha]-1, \quad j \in \mathbb{N},
\end{array}\right.
$$

where $\mathbb{N}$ and [.] denote the set of positive integers and the integer part function, respectively. Observe that IVP (1) refers to two different IVPs by a compact notation. If $\alpha \in] 0,1]$, the IVP (1) just has got the initial condition $Y(0)=\beta_{0}$, while if $\left.\left.\alpha \in\right] 1,2\right]$, the IVP (1) has got two initial conditions, $Y(0)=\beta_{0}$ and $Y^{\prime}(0)=\beta_{1}$. Henceforth, we will assume that input data $\gamma$ and $\lambda$ are independent real random variables defined in the Hilbert space $\left(\mathrm{L}^{2}(\Omega),\|\cdot\|_{2}\right)$ of second-order real random variables given by

$$
\mathrm{L}^{2}(\Omega)=\left\{X: \Omega \longrightarrow \mathbb{R}:\left(\mathbb{E}\left[X^{2}\right]\right)^{1 / 2}<+\infty\right\}, \quad\|X\|_{2}=\left(\mathbb{E}\left[X^{2}\right]\right)^{1 / 2}
$$

${ }_{44}$ The norm $\|\cdot\|_{2}$, usually referred to as 2-norm, is inferred from the inner product $\langle X, Y\rangle=\mathbb{E}[X Y]$, 
$X, Y \in \mathrm{L}^{2}(\Omega)$, being $\mathbb{E}[\cdot]$ the expectation operator. As usual, $(\Omega, \mathcal{F}, \mathbb{P})$ denotes a common underlying complete probability space for $\beta_{0}, \beta_{1}, \gamma$ and $\lambda$. Notice that every random variable with finite variance belongs to $\mathrm{L}^{2}(\Omega)$. This class of random variables is met in the most part of physical problems involving randomness. Given $\mathcal{T} \subset \mathbb{R}$, if $Y(t) \equiv\{Y(t): t \in \mathcal{T}\}$ is a second-order random variable for every $t \in \mathcal{T}$, then $Y(t)$ is termed a second-order stochastic process. The convergence inferred by the 2-norm is referred to as mean square convergence. Unless otherwise indicated, throughout this paper we will consider second-order random variables and secondorder stochastic processes.

In the recent contribution [19] by the authors, we have introduced the random mean square Caputo fractional derivative, $\left({ }^{C} D_{a^{+}}^{\alpha} Y\right)(t)$, of a second-order stochastic process $Y(t)$. Furthermore, we have constructed a mean square convergent random generalized power series solution to the random IVP (1) in the case that the order of the fractional derivative lies on the shorter interval, $0<\alpha \leq 1$. These results were established assuming the following conditions:

H1: Inputs $\beta_{0}, \gamma$ and $\lambda$ are (mutually) independent second-order random variables and,

H2: There exist constants $L>0$ and $H>0$ and an integer $m_{0}$ such that moments of random variable $\lambda$ satisfy

$$
\left\|\lambda^{m}\right\|_{2} \leq \sqrt{L} H^{m}<+\infty, \quad \forall m \text { integer }: m \geq m_{0} \geq 1 .
$$

In [19], the set of random variables satisfying latter condition are said to make up the class $\mathfrak{C}$. In [19], inequality (3) is derived from the condition that the absolute moments with respect to to the origin of the diffusion coefficient $\lambda$ growths exponentially, i.e., using Landau's notation there exist a constant $H>0$ and an integer $m_{0}$ such that

$$
\mathbb{E}\left[|\lambda|^{m}\right]=O\left(H^{m}\right), \quad \forall m \text { integer }: m \geq m_{0} \geq 1 .
$$

Furthermore, taking advantage of the following key result related to mean square convergence, approximations of the mean, $\mathbb{E}[Y(t)]$, and of the variance, $\mathbb{V}[Y(t)]$, of the solution stochastic process $Y(t)$ are also computed in [19].

Proposition 1. [3, Th. 4.4.3] Let $\left\{X_{M}: M \geq 0\right\}$ and $\left\{Z_{N}: N \geq 0\right\}$ be two sequences of secondorder random variables such that they are mean square convergent to $X$ and $Z$, respectively,

$$
X_{M} \underset{M \rightarrow+\infty}{\stackrel{m . s .}{\longrightarrow}} X, \quad Z_{N} \underset{N \rightarrow+\infty}{\stackrel{m . s .}{\longrightarrow}} Z \text {. }
$$

Then,

$$
\mathbb{E}\left[X_{M} Z_{N}\right] \underset{M, N \rightarrow+\infty}{\longrightarrow} \mathbb{E}[X Z]
$$

As a consequence of this previous result one gets

Corollary 1. Let $\left\{X_{M}: M \geq 0\right\}$ be a sequence of second-order random variables so that is mean square convergent to $X$, i.e., $X_{M} \underset{M \rightarrow+\infty}{\stackrel{m . s .}{\rightarrow}} X$. Then,

$$
\mathbb{E}\left[X_{M}\right] \underset{M \rightarrow+\infty}{\longrightarrow} \mathbb{E}[X] \text { and } \mathbb{V}\left[X_{M}\right] \underset{M \rightarrow+\infty}{\longrightarrow} \mathbb{V}[X]
$$


As it is indicated in Remark 6 of [19], hypothesis $\mathbf{H 2}$ is fulfilled for bounded random variables. Hence, the results established in [19] are applicable when the role of random input $\lambda$ is played by random variables such as, Binomial, Hypergeometric, Uniform, Trapezoidal, Beta, etc. Unfortunately, important unbounded random variables, such as Poisson, Exponential, or Gaussian random variables fail to satisfy hypothesis H2. To overcome this drawback, in [19] one proposes to use the so-called truncation technique [20, ch.V]. This approach permits to approximate unbounded random variables, say $X$, by bounded random variables, $\hat{X}$, resulting from the truncation of $X$. In this manner, random variable $\hat{X}$ is bounded and thereby hypothesis $\mathbf{H 2}$ is met. Nevertheless, if in the random fractional IVP (1) $\lambda$ input is an unbounded random variable, say a mean-zero Gaussian random variable with arbitrary variance $\sigma^{2}>0$, then under the approach proposed in [19], the original problem is not really addressed but approximating. As a consequence, approximation errors coming from the truncation procedure are introduced. Motivated by the previous exposition, in this paper we improve the results established in [19]. First, we will study the random general linear fractional differential equation in the case that the order of the fractional derivative $\alpha$ lies on the larger interval ]0,2] instead of assuming $0<\alpha \leq 1$. We point out that if $\alpha$ lies on an interval of the form $1<\alpha \leq 2$, then two initial conditions must be handled and the construction of the random generalized power series requires a more intricate process. Secondly, we will propose an alternative condition to hypothesis $\mathbf{H 2}$, which involves the $\lambda$ random input. As it shall be seen later, this new condition permits the consideration of important unbounded random variables, such that Gaussian and Exponential, avoiding the introduction of errors coming from the application of truncation technique. Furthermore, it shall be demonstrated that the random generalized power series (17) is still mean square convergent under our new hypotheses. Then according to Corollary 1, expressions (46) and (47) can be applied to compute reliable approximations for both the mean and the variance of the solution stochastic process $Y(t)$ to the random fractional IVP (1) with $\alpha_{0}=1$. Additionally, it is important to stress that the new condition is also satisfied by bounded random variables, thus the results established in [19] are fully retained in this new contribution.

The paper is organized as follows. In Section 2 we introduce a class of random variables that will play the role of diffusion coefficient $\lambda$ in the random IVP (1). By means of different examples, we show that this class contains all bounded random variables and significant unbounded random variables as well. The solution stochastic process to random IVP (1) is constructed by a random generalized power series whose mean square convergence is studied in Section 3. This analysis is divided in two cases depending of the order of the fractional derivative $\alpha$ : Case I corresponds to $\alpha \in] 0,1]$ while Case II deals with $\alpha \in] 1,2]$. In Section 4 we show several examples where our main theoretical findings are illustrated. Conclusions are drawn in Section 5.

\section{Introducing a key class of random variables}

In the next section, we shall construct a random generalized power series solution to IVP (1). This section is devoted to introduce a class of random variables that will allow us to enlarge, with respect to our previous contribution [19], the family of input data playing the role of the diffusion coefficient $\lambda$ in the IVP (1) for which the random generalized power series solution is mean square convergent.

Hereinafter we will assume that $\lambda$ is a second-order random variable such that

$$
\exists \eta, H>0, \quad p \geq 0:\left\|\lambda^{m}\right\|_{2} \leq \eta H^{m-1}((m-1) !)^{p}, \quad \forall m: m \geq m_{0} \geq 1, \quad m, m_{0} \text { integers. }
$$


The class of all random variables satisfying condition (4) will be denoted by $\hat{\mathfrak{C}}$. Observe that the latter condition contains as a particular case condition (3), since it is obtained by taking $p=0$ and $\eta=L / H>0$ in (4), i.e., $\mathfrak{C} \subset \hat{\mathfrak{C}}$. As a consequence, the results that will be presented in this contribution generalize the ones given in [19].

As it will be seen later, condition (4) is very useful to prove the mean square convergence of the random generalized power series to be constructed, however it may not be easy to check whether it is satisfied by specific families of random variables. This is the reason why we now introduce the following condition (5) that, in practice, is easier to check than (4) and, as it will be shown below, it entails condition (4). Motivated by this fact, let us assume that $\lambda$ is a secondorder random variable such that

$$
\exists p \geq 0: \frac{\left\|\lambda^{m+1}\right\|_{2}}{\left\|\lambda^{m}\right\|_{2}}=O\left(m^{p}\right), \quad \forall m: m \geq m_{0} \geq 1, \quad m, m_{0} \text { integers, }
$$

where $O(\cdot)$ denotes the Landau's symbol. By definition of $O(\cdot)$, condition (5) means

$$
\exists H, p \geq 0:\left\|\lambda^{m+1}\right\|_{2} \leq H m^{p}\left\|\lambda^{m}\right\|_{2}, \quad \forall m: m \geq m_{0} \geq 1, \quad m, m_{0} \text { integers. }
$$

Observe that it is sufficient this inequality to be fulfilled for $m_{0}$ large enough. As $\lambda \in \mathrm{L}^{2}(\Omega)$, $\|\lambda\|_{2}<+\infty$ and let $\eta$ be a finite positive number so that $\eta \geq\|\lambda\|_{2}$. Without loss of generality, hereinafter let us assume that $m_{0}=1$. Then, using a recursive argument in (6) one gets

$$
\begin{aligned}
\left\|\lambda^{m+1}\right\|_{2} & \leq H m^{p}\left\|\lambda^{m}\right\|_{2} \\
& \leq H^{2}(m(m-1))^{p}\left\|\lambda^{m-1}\right\|_{2} \\
& \leq H^{3}(m(m-1)(m-2))^{p}\left\|\lambda^{m-2}\right\|_{2} \\
& \vdots \\
& \leq \eta H^{m}(m !)^{p}, \quad \forall m \geq 1 \text { integer. }
\end{aligned}
$$

Summarizing, condition (5) (or equivalently, (6)) entails

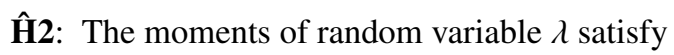

$$
\exists \eta, H>0, p \geq 0:\left\|\lambda^{m}\right\|_{2} \leq \eta H^{m-1}((m-1) !)^{p}, \quad \forall m \geq 1 \text { integer, }
$$

being $\eta \geq\|\lambda\|_{2}$ finite. Now, we introduce important families of random variables satisfying condition (5) (or equivalently (6)) and hence condition (7) too. It is important to highlight that such families correspond to both bounded and unbounded random variables.

Example 1. Let $\lambda$ be a bounded random variable. Then there exist real constants $l_{1}$ and $l_{2}$ with $l_{1}<l_{2}$ such that $\mathbb{P}\left[\left\{\omega \in \Omega: l_{1}(\omega)<\lambda(\omega) \leq l_{2}(\omega)\right\}\right]=1$. Observe that clearly $\lambda$ is a second-order random variable, i.e. $\lambda \in \mathrm{L}^{2}(\Omega)$. Let us assume, without loss of generality, that $\lambda$ is an absolutely continuous random variable being $f_{\lambda}(\lambda)$ its probability density function. If $\hat{l}=\max \left\{1,\left|l_{1}\right|,\left|l_{2}\right|\right\} \geq$ 1 , then

$$
\left\|\lambda^{m}\right\|_{2}=\left(\mathbb{E}\left[\lambda^{2 m}\right]\right)^{1 / 2}=\left(\int_{l_{1}}^{l_{2}} \lambda^{2 m} f_{\lambda}(\lambda) \mathrm{d} \lambda\right)^{1 / 2} \leq \hat{l}^{m}\left(\int_{l_{1}}^{l_{2}} f_{\lambda}(\lambda) \mathrm{d} \lambda\right)^{1 / 2}=\hat{l}^{m},
$$

where in the last step we have applied that $\int_{l_{1}}^{l_{2}} f_{\lambda}(\lambda) \mathrm{d} \lambda=1$ since $f_{\lambda}(\lambda)$ is a probability density function. Therefore, (7) is satisfied for $\eta=\hat{l}, H=\hat{l}^{m-1}$ and $p=0$. If $\hat{l}=\max \left\{\left|l_{1}\right|,\left|l_{2}\right|\right\} \leq 1$ 
instead, it is clear that $\left\|\lambda^{m}\right\|_{2} \leq 1$ and taking $\eta=H=1$ and $p=0$, condition (7) also holds. The previous reasoning is also valid if $\lambda$ is a discrete random variable. As a consequence, any truncated random variable as well as important bounded random variables such as Binomial, Hypergeometric, Uniform, Beta, Triangular, etc., satisfy condition (7).

Example 2. Let $\lambda$ be a Gaussian random variable with zero mean, $\mu=0$, and arbitrary finite variance, $\sigma^{2}>0$, i.e. $\lambda \sim N\left(0 ; \sigma^{2}\right)$. Hence, $\lambda \in \mathrm{L}^{2}(\Omega)$. It is known that (see [21], for instance)

$$
\mathbb{E}\left[\lambda^{n}\right]=\left\{\begin{array}{cc}
\frac{n !}{2^{\frac{n}{2}}\left(\frac{n}{2}\right) !} \sigma^{n} & \text { if } n \text { is even, } \\
0 & \text { if } n \text { is odd }
\end{array}\right.
$$

then, by the definition of the 2-norm (see (2)) one gets

$$
\frac{\left\|\lambda^{m+1}\right\|_{2}}{\left\|\lambda^{m}\right\|_{2}}=\frac{\left(\mathbb{E}\left[\lambda^{2(m+1)}\right]\right)^{1 / 2}}{\left(\mathbb{E}\left[\lambda^{2 m}\right]\right)^{1 / 2}}=\sigma \sqrt{\frac{(2 m+2)(2 m+1)}{2(m+1)}}=O\left(m^{1 / 2}\right) .
$$

Therefore, condition (5) is satisfied for $p=1 / 2$. Following the reasoning exhibited to deduce condition (7), it is straightforward to derive that this condition is fulfilled for $H=\sigma \sqrt{2}, p=1 / 2$ and $\eta=\sigma>0$.

Example 3. Let $\lambda$ be an Exponential random variable of parameter, $v>0$, i.e. $\lambda \sim \operatorname{Exp}(v)$. Hence, $\lambda \in \mathrm{L}^{2}(\Omega)$. It is known that (see [21], for instance)

$$
\mathbb{E}\left[\lambda^{m}\right]=\frac{m !}{v^{m}}, \quad m \geq 0,
$$

then

$$
\frac{\left\|\lambda^{m+1}\right\|_{2}}{\left\|\lambda^{m}\right\|_{2}}=\frac{\left(\mathbb{E}\left[\lambda^{2(m+1)}\right]\right)^{1 / 2}}{\left(\mathbb{E}\left[\lambda^{2 m}\right]\right)^{1 / 2}}=\frac{1}{v} \sqrt{(2 m+2)(2 m+1)}=O(m) .
$$

Therefore, condition (5) is satisfied for $p=1$. Moreover, condition (7) holds for $H=2 / v, p=1$ and $\eta=\sqrt{2} / v>0$.

Example 4. Let $\lambda$ be a Weibull random variable of parameters $a>0$ and $b>0$, i.e. $\lambda \sim$ $W e(a ; b)$. It is known that (see [21], for instance)

$$
\mathbb{E}\left[\lambda^{m}\right]=a^{m} \Gamma\left(1+\frac{m}{b}\right), \quad m \geq 0,
$$

159 being $\Gamma(\cdot)$ the classical gamma function. Using the definition of the 2-norm and (13), one gets

$$
\frac{\left\|\lambda^{m+1}\right\|_{2}}{\left\|\lambda^{m}\right\|_{2}}=\frac{\left(\mathbb{E}\left[\lambda^{2(m+1)}\right]\right)^{1 / 2}}{\left(\mathbb{E}\left[\lambda^{2 m}\right]\right)^{1 / 2}}=a \sqrt{\frac{\Gamma\left(1+\frac{2 m+2}{b}\right)}{\Gamma\left(1+\frac{2 m}{b}\right)}} .
$$

As condition (5) must be satisfied for $m \geq m_{0} \geq 1$ integer, then taking $m_{0}$ large enough and using Stirling's formula

$$
\Gamma(x+1) \approx x^{x} \mathrm{e}^{-x} \sqrt{2 \pi x}, \quad x \rightarrow+\infty,
$$




$$
\frac{\Gamma\left(1+\frac{2(m+1)}{b}\right)}{\Gamma\left(1+\frac{2 m}{b}\right)} \approx \frac{\left(\frac{2(m+1)}{b}\right)^{\frac{2(m+1)}{b}} \mathrm{e}^{-\frac{2(m+1)}{b}} \sqrt{2 \pi \frac{2(m+1)}{b}}}{\left(\frac{2 m}{b}\right)^{\frac{2 m}{b}} \mathrm{e}^{-\frac{2 m}{b}} \sqrt{2 \pi \frac{2 m}{b}}} \approx\left(\frac{m+1}{m}\right)^{\frac{2 m}{b}}\left(\frac{2(m+1)}{b}\right)^{\frac{2}{b}} e^{-\frac{2}{b}} \sqrt{\frac{m+1}{m}} \approx\left(\frac{2 m}{b}\right)^{\frac{2}{b}},
$$

where in the last step we have used that $\left(\frac{m+1}{m}\right)^{\frac{2 m}{b}} \stackrel{m \rightarrow+\infty}{\longrightarrow} \mathrm{e}^{\frac{2}{b}}$. Then, substituting (16) in (14) one deduces

$$
\frac{\left\|\lambda^{m+1}\right\|_{2}}{\left\|\lambda^{m}\right\|_{2}} \approx a \sqrt{\left(\frac{2 m}{b}\right)^{2 / b}}=O\left(m^{\frac{1}{b}}\right) \text {. }
$$

As a consequence, $\lambda \sim$ We $(a ; b)$ satisfies condition (5) for $p=1 / b>0$ and also, condition (7) and (6) are satisfied taking $H=a(2 / b)^{1 / b}$ and $\eta=a \sqrt{\Gamma(1+2 / b)}$.

\section{Mean square convergence of the random generalized power series solution}

This section is devoted to construct a random generalized power series solution to the IVP (1) and then proving its mean square convergence. Finally, we will give closed-form expressions for the approximations of the mean, the variance and the covariance functions of the solution stochastic process.

The analysis will be split in two cases: Case I corresponding to $0<\alpha \leq 1$ and Case II corresponding to $1<\alpha \leq 2$. The former is strongly related to the results established in [19], hence it will be discussed taking advantage of such previous findings. In particular, as the representation of the solution stochastic process is just the one shown in [19], here we will focus on the analysis of the mean square convergence assuming that the diffusion coefficient $\lambda$ satisfies condition $\hat{\mathbf{H}} 2$ (see expression (4)) instead of $\mathbf{H 2}$ (see expression (3)). As Case II involves the two random variables $\beta_{0}$ and $\beta_{1}$ through initial conditions, it will be assumed the following hypothesis:

H1: Inputs $\beta_{0}, \beta_{1}, \gamma$ and $\lambda$ are (mutually) independent second-order random variables,

instead of H1. As it shall be seen later, the study of Case II will require further analysis.

\subsection{Case I: $0<\alpha \leq 1$}

In accordance with [19], it is known that the solution stochastic process to the random fractional IVP (1) $0<\alpha \leq 1$ is given by

$$
Y(t)=\sum_{m=0}^{+\infty} \frac{\lambda^{m} \beta_{0}}{\Gamma(\alpha m+1)} t^{\alpha m}+\sum_{m=1}^{+\infty} \frac{\lambda^{m-1} \gamma}{\Gamma(\alpha m+1)} t^{\alpha m}
$$

In this section we will establish sufficient conditions in order to guarantee the mean square convergence of this random generalized power series assuming that input data $\beta_{0}, \gamma$ and $\lambda$ satisfy hypotheses $\mathbf{H 1}$ and $\hat{\mathbf{H}} \mathbf{2}$. This will be done just for the first series in (17), since the proof for the second series can be done analogously.

Let us observe that for $0<\alpha \leq 1$ and $t>0$ one gets

$$
\left\|\frac{\lambda^{m} \beta_{0}}{\Gamma(\alpha m+1)} t^{\alpha m}\right\|_{2}=\frac{\left\|\lambda^{m}\right\|_{2}\left\|\beta_{0}\right\|_{2}}{\Gamma(\alpha m+1)} t^{\alpha m} \leq \frac{\eta H^{m-1}((m-1) !)^{p}\left\|\beta_{0}\right\|_{2}}{\Gamma(\alpha m+1)} t^{\alpha m}=: \delta_{m}(t),
$$


where probabilistic independence between random variables $\lambda^{m}$ and $\beta_{0}$ (justified by hypothesis H1 and Propostion 2 of [19], see also [22, p.92]) and hypothesis $\hat{\mathbf{H}} \mathbf{2}$ have been applied. Down below, we obtain sufficient conditions for the mean square absolute convergence of first series in (17) using the D'Alembert or ratio test

$$
\begin{aligned}
\lim _{m \rightarrow+\infty} \frac{\delta_{m+1}(t)}{\delta_{m}(t)} & =H t^{\alpha} \lim _{m \rightarrow+\infty}\left(m^{p} \frac{\Gamma(\alpha m+1)}{\Gamma(\alpha(m+1)+1)}\right) \\
& =H\left(\frac{t}{\alpha}\right)^{\alpha} \lim _{m \rightarrow+\infty} \frac{m^{p}}{(m+1)^{\alpha}} \\
& =\left\{\begin{array}{cl}
0 & \text { if } 0 \leq p<\alpha, \forall t>0, \\
H\left(\frac{t}{\alpha}\right)^{\alpha} & \text { if } 0 \leq p=\alpha, \forall t>0 .
\end{array}\right.
\end{aligned}
$$

Observe that in the second earlier step we have used the Stirling's formula (15) to conclude

$$
\lim _{m \rightarrow+\infty} \frac{\Gamma(\alpha m+1)}{\Gamma(\alpha(m+1)+1)}=\lim _{m \rightarrow+\infty} \frac{(\alpha m)^{\alpha m} \mathrm{e}^{-\alpha m} \sqrt{2 \pi \alpha m}}{(\alpha(m+1))^{\alpha(m+1)} \mathrm{e}^{-\alpha(m+1)} \sqrt{2 \pi \alpha(m+1)}}=\frac{1}{\alpha^{\alpha}} \lim _{m \rightarrow+\infty} \frac{1}{(m+1)^{\alpha}} .
$$

As a consequence of the previous development together with Corollary 1, the following result has been established

Theorem 1. Let us consider the random fractional IVP (1) with $0<\alpha \leq 1$ and assume that the inputs data $\beta_{0}, \gamma$ and $\lambda$ are random variables satisfying hypotheses $\mathbf{H 1}$ and $\hat{\mathbf{H} 2}$. If $p \geq 0$ and $\alpha \in] 0,1]$ are so that $p<\alpha$, then the random generalized power series $Y(t)$ given by (17) is a mean square solution to the IVP (1) for all $t \geq 0$. While, if $p=\alpha$, then $Y(t)$ is a mean square solution to the IVP (1) over the domain $t: 0 \leq t<\alpha / H^{\frac{1}{\alpha}}$. Furthermore, the approximations of the mean and the variance (or standard deviation) given by (46) and (47) (see Appendix I), respectively, will also converge at least over the domains previously specified for the mean square convergence.

Remark 1. The rigorous construction of solution stochastic process (17) would require to check some technical hypotheses. This analysis has been omitted here because it follows an analogous development to one exhibited in [19], but using the new hypothesis $\hat{\mathbf{H}} \mathbf{2}$ for $\lambda$ instead of $\mathbf{H 2}$.

Remark 2. The above result provides sufficient conditions to guarantee the mean square convergence of the random generalized power series solution (17) to the random fractional IVP (1) assuming mild hypotheses that include a wide range of random variables, namely all bounded random variables and significant unbounded random variables such as Gaussian and Weibull, for instance. It is interesting to observe that our mean square convergence analysis depends on parameter $p$ associated to the diffusion random variable $\lambda$ (see expression (7)) and on the order of the fractional derivative $\alpha \in] 0,1]$. In Th. 1 we have shown that the random generalized power series (17) is mean square unconditionally convergent for all $t \geq 0$ provided $p<\alpha$, while the domain of mean square convergence becomes smaller when $p=\alpha$, specifically $t: 0 \leq t<\alpha / H^{\frac{1}{\alpha}}$. Thus, in this latter case the domain depends on both the constant $H$ associated to hypothesis $\hat{\mathbf{H}} 2$ (see expression (7)) and on the order of the fractional derivative $\alpha \in] 0,1]$. This issue will be analyzed deeper throughout the examples exhibited in the next section.

\subsection{Case II: $1<\alpha \leq 2$}

This section is devoted to construct a solution stochastic process to random IVP (1) when $1<\alpha \leq 2$. This solution is then constructed by means of a random generalized power series. 
We will prove the mean square convergence of this series under mild conditions. Finally, we will provide approximations of the mean, the variance, the covariance and the cross-covariance function of the solution stochastic process.

The solution stochastic process will be sought by combining the random Fröbenius method and a mean square chain rule for differentiating second-order stochastic processes, that has been recently established by the authors [23]. As our subsequent development follows in broad outline that of ideas exhibited in [19], it will be presented in a direct manner. The solution stochastic process $Y(t)$ will be constructed in the following form

$$
Y(t)=Y_{1}(t)+Y_{2}(t), \quad\left\{\begin{array}{l}
Y_{1}(t)=\sum_{m \geq 0} X_{m} t^{\alpha m}, \\
Y_{2}(t)=\sum_{m \geq 0} Y_{m} t^{\alpha m+1} .
\end{array}\right.
$$

In order to apply Fröbenius method, first we need to obtain the mean square Caputo derivative of order $\alpha$ to $Y_{1}(t)$ and $Y_{2}(t)$. To this end, we define $\hat{Y}_{1}(t)=\sum_{m \geq 0} X_{m} t^{m}$, hence $Y_{1}(t)=\hat{Y}_{1}\left(t^{\alpha}\right)$. According to [19], the random mean square Caputo derivative is given by

$$
\left({ }^{C} D_{0^{+}}^{\alpha} Y_{1}\right)(t)=\left({ }^{C} D_{0^{+}}^{\alpha} \hat{Y}_{1}\right)\left(t^{\alpha}\right)=\left(J_{0^{+}}^{2-\alpha} Z\right)(t), \quad 1<\alpha \leq 2,
$$

C2: $\hat{Y}_{1}^{\prime}(t)$ is a mean square differentiable at $v=t^{\alpha}$. Moreover,

$$
\hat{Y}_{1}^{\prime \prime}\left(t^{\alpha}\right)=\sum_{m \geq 2} m(m-1) X_{m} t^{\alpha(m-2)} .
$$

C3: $\frac{\mathrm{d} \hat{Y}_{1}(v)}{\mathrm{d} v}$ is mean square continuous on $\left.v \in\right] 0,+\infty[$.

C4: $\frac{\mathrm{d}^{2} \hat{Y}_{1}(v)}{\mathrm{d}^{2} v}$ is mean square continuous on $\left.v \in\right] 0,+\infty[$.

In that case

$$
\begin{aligned}
Z(t) & =\left[\left(\hat{Y}_{1}\left(t^{\alpha}\right)\right)^{\prime}\right]^{\prime}=\left[\left.\alpha t^{\alpha-1} \hat{Y}_{1}^{\prime}(v)\right|_{v=t^{\alpha}}\right]^{\prime} \\
& =\left.\alpha(\alpha-1) t^{\alpha-2} \hat{Y}_{1}^{\prime}(v)\right|_{v=t^{\alpha}}+\left.\alpha t^{\alpha-1} \alpha t^{\alpha-1} \hat{Y}_{1}^{\prime \prime}(v)\right|_{v=t^{\alpha}} \\
& =\left.\alpha(\alpha-1) t^{\alpha-2} \hat{Y}_{1}^{\prime}(v)\right|_{v=t^{\alpha}}+\left.\alpha^{2} t^{2 \alpha-2} \hat{Y}_{1}^{\prime \prime}(v)\right|_{v=t^{\alpha}}\left(\sum_{m \geq 0}(m+1) X_{m+1} t^{\alpha(m+1)-2}+\alpha^{2} \sum_{m \geq 0}(m+2)(m+1) X_{m+2} t^{\alpha(m+2)-2} .\right. \\
& =\alpha(\alpha-1) \sum .
\end{aligned}
$$


241

242

Observe that, we have applied Property (4.126) of [3, p.96] to compute the mean square derivative of the product of the deterministic function $\alpha t^{\alpha-1}$ and the second-order stochastic process $\hat{Y}_{1}^{\prime}\left(t^{\alpha}\right)$.

In order to legitimate the computation of the mean square Caputo derivative $\left({ }^{C} D_{0^{+}}^{\alpha} Y_{1}\right)(t)$, we further assume that the following conditions

C5: The random generalized power series $\sum_{m \geq 0}(m+1) X_{m+1} t^{\alpha(m+1)-2}$ is mean square uniformly convergent on $t>0$,

C6: The random generalized power series $\sum_{m \geq 0}(m+2)(m+1) X_{m+2} t^{\alpha(m+2)-2}$ is mean square uniformly convergent on $t>0$, 
hold. Then,

$$
\begin{aligned}
& \left({ }^{C} D_{0^{+}}^{\alpha} Y_{1}\right)(t)=\left(J_{0^{+}}^{2-\alpha} Z\right)(t) \\
& =J_{0^{+}}^{2-\alpha}\left(\alpha(\alpha-1) \sum_{m \geq 0}(m+1) X_{m+1} t^{\alpha(m+1)-2}+\alpha^{2} \sum_{m \geq 0}(m+2)(m+1) X_{m+2} t^{\alpha(m+2)-2}\right) \\
& =\alpha(\alpha-1) \sum_{m \geq 0}(m+1) X_{m+1} J_{0^{+}}^{2-\alpha}\left(t^{\alpha(m+1)-2}\right)+\alpha^{2} \sum_{m \geq 0}(m+2)(m+1) X_{m+2} J_{0^{+}}^{2-\alpha}\left(t^{\alpha(m+2)-2}\right) \\
& =\alpha(\alpha-1) \sum_{m \geq 0}(m+1) X_{m+1}\left(\frac{1}{\Gamma(2-\alpha)} \int_{0}^{t}(t-u)^{1-\alpha} u^{\alpha(m+1)-2} \mathrm{~d} u\right) \\
& +\alpha^{2} \sum_{m \geq 0}(m+2)(m+1) X_{m+2}\left(\frac{1}{\Gamma(2-\alpha)} \int_{0}^{t}(t-u)^{1-\alpha} u^{\alpha(m+2)-2} \mathrm{~d} u\right) \\
& =\alpha(\alpha-1) \sum_{m \geq 0}(m+1) \frac{\Gamma(\alpha(m+1)-1)}{\Gamma(\alpha m+1)} X_{m+1} t^{\alpha m} \\
& +\alpha^{2} \sum_{m \geq 0}(m+2)(m+1) \frac{\Gamma(\alpha(m+2)-1)}{\Gamma(\alpha(m+1)+1)} X_{m+2} t^{\alpha(m+1)} \\
& =\alpha(\alpha-1) \Gamma(\alpha-1) X_{1}+\sum_{m \geq 1} \alpha(\alpha-1)(m+1) \frac{\Gamma(\alpha(m+1)-1)}{\Gamma(\alpha m+1)} X_{m+1} t^{\alpha m} \\
& +\alpha^{2} \sum_{m \geq 0}(m+2)(m+1) \frac{\Gamma(\alpha(m+2)-1)}{\Gamma(\alpha(m+1)+1)} X_{m+2} t^{\alpha(m+1)} \\
& =\Gamma(\alpha+1) X_{1}+\sum_{m \geq 0} \alpha(\alpha-1)(m+2) \frac{\Gamma(\alpha(m+2)-1)}{\Gamma(\alpha(m+1)+1)} X_{m+2} t^{\alpha(m+1)} \\
& +\sum_{m \geq 0} \alpha^{2}(m+2)(m+1) \frac{\Gamma(\alpha(m+2)-1)}{\Gamma(\alpha(m+1)+1)} X_{m+2} t^{\alpha(m+1)} \\
& =\Gamma(\alpha+1) X_{1}+\sum_{m \geq 0}(\alpha-1+\alpha(m+1)) \alpha(m+2) \frac{\Gamma(\alpha(m+2)-1)}{\Gamma(\alpha(m+1)+1)} X_{m+2} t^{\alpha(m+1)} \\
& =\Gamma(\alpha+1) X_{1}+\sum_{m \geq 0}(\alpha(m+2)-1) \alpha(m+2) \frac{\Gamma(\alpha(m+2)-1)}{\Gamma(\alpha(m+1)+1)} X_{m+2} t^{\alpha(m+1)} \\
& =\Gamma(\alpha+1) X_{1}+\sum_{m \geq 0} y \frac{\Gamma(\alpha(m+2)+1)}{\Gamma(\alpha(m+1)+1)} X_{m+2} t^{\alpha(m+1)} \\
& =\Gamma(\alpha+1) X_{1}+\sum_{m \geq 1} \frac{\Gamma(\alpha(m+1)+1)}{\Gamma(\alpha m+1)} X_{m+1} t^{\alpha m} \\
& =\sum_{m \geq 0} \frac{\Gamma(\alpha(m+1)+1)}{\Gamma(\alpha m+1)} X_{m+1} t^{\alpha m} \text {, }
\end{aligned}
$$

where we have used the reproductive property of gamma function, $\Gamma(\gamma+1)=\gamma \Gamma(\gamma), \gamma>0$.

Now, we compute the random mean square Caputo derivative of $Y_{2}(t)$. Note that by the definition of mean square Caputo derivative (see [19]) one gets

$$
\left({ }^{C} D_{0^{+}}^{\alpha} Y_{2}\right)(t)=\left(J_{0^{+}}^{2-\alpha} Y_{2}^{\prime \prime}\right)(t)=\left(J_{0^{+}}^{2-\alpha}\left(Y_{2}^{\prime}\right)^{\prime}\right)(t)=\left({ }^{C} D_{0^{+}}^{\alpha-1} Y_{2}^{\prime}\right)(t)
$$


As $1<\alpha \leq 2$, and $Y_{2}^{\prime}(t)=\sum_{m \geq 0}(\alpha m+1) Y_{m} t^{\alpha m}$, we can recast $\left.\left.\hat{\alpha}=\alpha-1 \in\right] 0,1\right], \hat{Y}_{m}=(\alpha m+1) Y_{m}$ and compute the random mean square Caputo derivative of order $\hat{\alpha}$ of $\sum_{m \geq 0} \hat{Y}_{m} t^{\alpha m}$. Using the same argument shown in [19] (see expression (25)) one obtains

$$
\left({ }^{C} D_{0^{+}}^{\alpha} Y_{2}\right)(t)=\sum_{m \geq 0} Y_{m+1} \frac{\Gamma(\alpha(m+1)+2)}{\Gamma(\alpha m+2)} t^{\alpha m+1} .
$$

Once we have obtained the mean square Caputo derivative of both series given in (18), we need to compute their coefficients $X_{m}$ and $Y_{m}$. This can be done by substituting the expressions of the Caputo derivative of $Y_{1}(t)$ and $Y_{2}(t)$, given by (21) and (22), respectively, in random IVP (1) taking into account that $\left({ }^{C} D_{0^{+}}^{\alpha} Y\right)(t)=\left({ }^{C} D_{0^{+}}^{\alpha} Y_{1}\right)(t)+\left({ }^{C} D_{0^{+}}^{\alpha} Y_{2}\right)(t)$. This yields

$$
\sum_{m \geq 0} \frac{\Gamma(\alpha(m+1)+1)}{\Gamma(\alpha m+1)} X_{m+1} t^{\alpha m}-\lambda \sum_{m \geq 0} X_{m} t^{\alpha m}+\sum_{m \geq 0} \frac{\Gamma(\alpha(m+1)+2)}{\Gamma(\alpha m+2)} Y_{m+1} t^{\alpha m+1}-\lambda \sum_{m \geq 0} Y_{m} t^{\alpha m+1}=\gamma,
$$

thus

$\Gamma(\alpha+1) X_{1}-\lambda X_{0}+\sum_{m \geq 1}\left(\frac{\Gamma(\alpha(m+1)+1)}{\Gamma(\alpha m+1)} X_{m+1}-\lambda X_{m}\right) t^{\alpha m}+\sum_{m \geq 0}\left(\frac{\Gamma(\alpha(m+1)+2)}{\Gamma(\alpha m+2)} Y_{m+1}-\lambda Y_{m}\right) t^{\alpha m+1}=\gamma$.

259 If the following recurrences for coefficients $X_{m}$

$$
X_{1}=\frac{\lambda X_{0}+\gamma}{\Gamma(\alpha+1)}, \quad X_{m+1}=\frac{\lambda \Gamma(\alpha m+1)}{\Gamma(\alpha(m+1)+1)} X_{m}, \quad m \geq 1,
$$

and $Y_{m}$

$$
Y_{m+1}=\frac{\lambda \Gamma(\alpha m+2)}{\Gamma(\alpha(m+1)+2)} Y_{m}, \quad m \geq 0
$$

are satisfied, then it is guaranteed that the relationship (24) hold. Taking into account the initial conditions $Y(0)=X_{0}=\beta_{0}$ and $Y^{\prime}(0)=Y_{0}=\beta_{1}$, and using recurrences (25) and (26) one gets

$$
X_{m}=\frac{\lambda^{m} \beta_{0}+\lambda^{m-1} \gamma}{\Gamma(\alpha m+1)}, \quad Y_{m}=\frac{\lambda^{m} \beta_{1}}{\Gamma(\alpha m+2)}, \quad m \geq 1 .
$$

Therefore, a candidate solution stochastic process to the random IVP (1) with $1<\alpha \leq 2$ is given by

$$
Y(t)=\sum_{m \geq 0} X_{m, 1} t^{\alpha m}+\sum_{m \geq 1} X_{m, 2} t^{\alpha m}+\sum_{m \geq 0} Y_{m} t^{\alpha m+1}
$$

where

$$
X_{m, 1}=\frac{\lambda^{m} \beta_{0}}{\Gamma(\alpha m+1)}, \quad X_{m, 2}=\frac{\lambda^{m-1} \gamma}{\Gamma(\alpha m+1)}, \quad Y_{m}=\frac{\lambda^{m} \beta_{1}}{\Gamma(\alpha m+2)} .
$$

Observe that for convenience, the general term of series $X_{m} t^{\alpha m}$ has been split in two pieces. So far, we have constructed a formal solution stochastic process to random IVP (1) and now, assuming that input random variables satisfy hypotheses $\hat{\mathbf{H}} \mathbf{1}$ and $\hat{\mathbf{H}} \mathbf{2}$, we need to check that conditions C1-C6 hold. As this can be done by taking the same steps shown in detail in [19], they will be skipped here. The analysis of mean square convergence of (27)-(28) can be carried out as shown in Case I since the involved series are identical and/or very similar, hence we omit this discussion. 
To compute approximations for the mean of the solution stochastic process $Y(t)$, we first consider the truncation of order, say $M$, of the infinite series (27)-(28), i.e.,

$$
Y_{M}(t)=\sum_{m=0}^{M} X_{m, 1} t^{\alpha m}+\sum_{m=1}^{M} X_{m, 2} t^{\alpha m}+\sum_{m=0}^{M} Y_{m} t^{\alpha m+1}
$$

and then, we take the expectation operator. Using independence of $\beta_{0}, \beta_{1}, \gamma$ and $\lambda$ (see $\hat{\mathbf{H}} \mathbf{1}$ ), one obtains

$$
\mathbb{E}\left[Y_{M}(t)\right]=\mathbb{E}\left[\beta_{0}\right] \sum_{m=0}^{M} \frac{\mathbb{E}\left[\lambda^{m}\right]}{\Gamma(\alpha m+1)} t^{\alpha m}+\mathbb{E}[\gamma] \sum_{m=1}^{M} \frac{\mathbb{E}\left[\lambda^{m-1}\right]}{\Gamma(\alpha m+1)} t^{\alpha m}+\mathbb{E}\left[\beta_{1}\right] \sum_{m=0}^{M} \frac{\mathbb{E}\left[\lambda^{m}\right]}{\Gamma(\alpha m+2)} t^{\alpha m+1}
$$

Instead of providing approximations for the variance (or standard deviation) function of $Y(t)$, we will give more general approximations. Indeed, our first step will obtain approximations of the cross-covariance function of $Y(t), \mathbb{C}_{Y_{M}, Y_{N}}(t, s)$, by considering two different truncations $Y_{M}(t)$ and $Y_{N}(s)$ at the points $t$ and $s$, respectively,

$$
\begin{aligned}
\mathbb{C}_{Y_{M}, Y_{N}}(t, s)= & \sum_{m=0}^{M} \sum_{n=0}^{N} \operatorname{Cov}\left[X_{m, 1}, X_{n, 1}\right] t^{\alpha m} s^{\alpha n}+\sum_{m=0}^{M} \sum_{n=1}^{N} \operatorname{Cov}\left[X_{m, 1}, X_{n, 2}\right] t^{\alpha m} s^{\alpha n} \\
& +\sum_{m=0}^{M} \sum_{n=0}^{N} \operatorname{Cov}\left[X_{m, 1}, Y_{n}\right] t^{\alpha m} s^{\alpha n+1}+\sum_{m=1}^{M} \sum_{n=0}^{N} \operatorname{Cov}\left[X_{m, 2}, X_{n, 1}\right] t^{\alpha m} s^{\alpha n} \\
& +\sum_{m=1}^{M} \sum_{n=1}^{N} \operatorname{Cov}\left[X_{m, 2}, X_{n, 2}\right] t^{\alpha m} s^{\alpha n}+\sum_{m=1}^{M} \sum_{n=0}^{N} \operatorname{Cov}\left[X_{m, 2}, Y_{n}\right] t^{\alpha m} s^{\alpha n+1} \\
& +\sum_{m=0}^{M} \sum_{n=0}^{N} \operatorname{Cov}\left[Y_{m}, Y_{n}\right] t^{\alpha m+1} s^{\alpha n+1}+\sum_{m=0}^{M} \sum_{n=1}^{N} \operatorname{Cov}\left[Y_{m}, X_{n, 2}\right] t^{\alpha m+1} s^{\alpha n} \\
& +\sum_{m=0}^{M} \sum_{n=1}^{N} \operatorname{Cov}\left[Y_{m}, Y_{n}\right] t^{\alpha m+1} s^{\alpha n+1},
\end{aligned}
$$

where $\operatorname{Cov}[\cdot, \cdot]$ denotes the covariance operator. Applying hypothesis $\hat{\mathbf{H}} \mathbf{1}$, each covariance can 
be expressed in terms of data as follows

$$
\begin{aligned}
\operatorname{Cov}\left[X_{m, 1}, X_{n, 1}\right] & =\frac{\mathbb{E}\left[\lambda^{m+n}\right] \mathbb{E}\left[\left(\beta_{0}\right)^{2}\right]-\mathbb{E}\left[\lambda^{m}\right] \mathbb{E}\left[\lambda^{n}\right]\left(\mathbb{E}\left[\beta_{0}\right]\right)^{2}}{\Gamma(\alpha m+1) \Gamma(\alpha n+1)}, \\
\operatorname{Cov}\left[X_{m, 1}, X_{n, 2}\right] & =\frac{\left(\mathbb{E}\left[\lambda^{m+n-1}\right]-\mathbb{E}\left[\lambda^{m}\right] \mathbb{E}\left[\lambda^{n-1}\right]\right) \mathbb{E}\left[\beta_{0}\right] \mathbb{E}[\gamma]}{\Gamma(\alpha m+1) \Gamma(\alpha n+1)}, \\
\operatorname{Cov}\left[X_{m, 1}, Y_{n}\right] & =\frac{\left(\mathbb{E}\left[\lambda^{m+n}\right]-\mathbb{E}\left[\lambda^{m}\right] \mathbb{E}\left[\lambda^{n}\right]\right) \mathbb{E}\left[\beta_{0}\right] \mathbb{E}\left[\beta_{1}\right]}{\Gamma(\alpha m+1) \Gamma(\alpha n+2)}, \\
\operatorname{Cov}\left[X_{m, 2}, X_{n, 1}\right] & =\frac{\left(\mathbb{E}\left[\lambda^{m+n-1}\right]-\mathbb{E}\left[\lambda^{m-1}\right] \mathbb{E}\left[\lambda^{n}\right]\right) \mathbb{E}\left[\beta_{0}\right] \mathbb{E}[\gamma]}{\Gamma(\alpha m+1) \Gamma(\alpha n+1)} \\
\operatorname{Cov}\left[X_{m, 2}, X_{n, 2}\right] & =\frac{\mathbb{E}\left[\lambda^{m+n-2}\right] \mathbb{E}\left[\gamma^{2}\right]-\mathbb{E}\left[\lambda^{m-1}\right] \mathbb{E}\left[\lambda^{n-1}\right](\mathbb{E}[\gamma])^{2}}{\Gamma(\alpha m+1) \Gamma(\alpha n+1)}, \\
\operatorname{Cov}\left[X_{m, 2}, Y_{n}\right] & =\frac{\left(\mathbb{E}\left[\lambda^{m+n-1}\right]-\mathbb{E}\left[\lambda^{m-1}\right] \mathbb{E}\left[\lambda^{n}\right]\right) \mathbb{E}[\gamma] \mathbb{E}\left[\beta_{1}\right]}{\Gamma(\alpha m+1) \Gamma(\alpha n+2)}, \\
\operatorname{Cov}\left[Y_{m}, X_{n, 1}\right] & =\frac{\left(\mathbb{E}\left[\lambda^{m+n}\right]-\mathbb{E}\left[\lambda^{m}\right] \mathbb{E}\left[\lambda^{n}\right]\right) \mathbb{E}\left[\beta_{0}\right] \mathbb{E}\left[\beta_{1}\right]}{\Gamma(\alpha m+2) \Gamma(\alpha n+1)}, \\
\operatorname{Cov}\left[Y_{m}, X_{n, 2}\right] & =\frac{\left(\mathbb{E}\left[\lambda^{m+n-1}\right]-\mathbb{E}\left[\lambda^{m}\right] \mathbb{E}\left[\lambda^{n-1}\right]\right) \mathbb{E}[\gamma] \mathbb{E}\left[\beta_{1}\right]}{\Gamma(\alpha m+2) \Gamma(\alpha n+1)}, \\
\operatorname{Cov}\left[Y_{m}, Y_{n}\right] & =\frac{\mathbb{E}\left[\lambda^{m+n}\right] \mathbb{E}\left[\beta_{1}^{2}\right]-\mathbb{E}\left[\lambda^{m}\right] \mathbb{E}\left[\lambda^{n}\right]\left(\mathbb{E}\left[\beta_{1}\right]\right)^{2}}{\Gamma(\alpha m+2) \Gamma(\alpha n+1)} .
\end{aligned}
$$

If we take $M=N$ in (31), we then obtain the covariance function, $\mathbb{C}_{Y_{M}}(t, s)$, of the approximation $Y_{M}(t)$, while its variance function is derived taking $t=s$ in the covariance function, i.e.,

$$
\mathbb{C}_{Y_{M}}(t, s)=\mathbb{C}_{Y_{M}, Y_{M}}(t, s), \quad \mathbb{V}\left[Y_{M}(t)\right]=\mathbb{C}_{Y_{M}}(t, t) .
$$

Summarizing the following result has been established

Theorem 2. Let us consider the random fractional IVP (1) with $1<\alpha \leq 2$ and assume that the inputs data $\beta_{0}, \beta_{1}, \gamma$ and $\lambda$ are random variables satisfying hypotheses $\hat{\mathbf{H}} \mathbf{1}$ and $\hat{\mathbf{H}} 2$. If $p \geq 0$ and $\alpha \in] 1,2]$ are so that $p<\alpha$, then the random generalized power series $Y(t)$ given by (27)-(28) is a mean square solution to the IVP (1) for all $t \geq 0$. While, if $p=\alpha$, then $Y(t)$ is a mean square solution to the IVP (1) over the domain $t: 0 \leq t<\alpha / H^{\frac{1}{\alpha}}$. Furthermore, the approximations of the mean and the variance (or standard deviation) given by (30) and (31)-(41), respectively, will also converge at least over the domains previously specified for the mean square convergence.

Similar comments to the ones contained in Remark 2 can now be made with respect to the intervals of convergence to the mean and the variance determined in Th. 2 .

\section{Numerical examples}

This section is devoted to illustrate, through a variety of examples, the results established in Theorems 1 and 2. Particularly we investigate, through examples, if the domain of convergence of the mean of the solution stochastic process to the random fractional IVP (1) can be 
enlarger with respect the one inferred from the mean square convergence. This issue will be discussed through the approximations for statistical moments given in Section 3. The examples have been devised to take into consideration both bounded and unbounded random variables for the diffusion coefficient $\lambda$. In the examples, the accuracy of the approximations of the mean and standard deviation will be measured using the following relative errors (RE) between consecutive approximations of order $M$ and $M+1$, using different values of $M$ and different time instants $t$,

$$
\operatorname{RE}(\text { Mean })(t ; M)=\left|\frac{\mathbb{E}\left[Y_{M+1}(t)\right]-\mathbb{E}\left[Y_{M}(t)\right]}{\mathbb{E}\left[Y_{M}(t)\right]}\right|,
$$

$$
\operatorname{RE}(\operatorname{Sd})(t ; M)=\left|\frac{\sqrt{\mathbb{V}\left[Y_{M+1}(t)\right]}-\sqrt{\mathbb{V}\left[Y_{M}(t)\right]}}{\sqrt{\mathbb{V}\left[Y_{M}(t)\right]}}\right| .
$$

Here, $\mathbb{E}\left[Y_{M}(t)\right]$ and $\mathbb{V}\left[Y_{M}(t)\right]$ are given by expressions (46) and (47), in Case I, and by (31)-(41), in Case II, respectively.

Example 5. This example illustrates Case I, corresponding to $\alpha \in] 0,1]$, when diffusion coefficient $\lambda$ is a bounded random variable. Let us consider the random fractional IVP (1), where

- $\beta_{0}$ is an Exponential random variable of mean $1 / 5$ and variance $1 / 25$, i.e., $\beta_{0} \sim \operatorname{Exp}(5)$;

- $\gamma$ is a Gaussian random variable with zero mean and unit standard deviation, $\gamma \sim N(0 ; 1)$ and

- $\lambda$ is a Beta random variable of mean $2 / 5$ and variance $1 / 25, \lambda \sim B e(2 ; 3)$.

We will also assume that $\beta_{0}, \gamma$ and $\lambda$ are independent random variables. Since $\lambda$ is a bounded random variable (it lies on the interval ]0, 1[), by Example 1 we know that $\lambda$ satisfies hypothesis $\hat{\mathbf{H}}$ 2. Also, clearly all these input data are second-order random variables because they have finite variance. As a consequence, hypothesis $\hat{\mathbf{H}} \mathbf{1}$ also holds and Th. 1 can be applied. Observe that the parameter $p$ associated to $\lambda$ is $p=0$ (see Example 1). According to Th. 1 the solution $Y(t)$, given by (17), is mean square convergent for all $t \geq 0$. Therefore, the expectation and the variance (or equivalently, the standard deviation) of $Y(t)$, which are given by (46) and (47), respectively, will also converge for all $t \geq 0$, independently of the order $\alpha \in] 0,1]$ of the fractional derivative. This conclusion is illustrated in Fig. $1(\alpha=0.3)$ and in Fig. $2(\alpha=0.7)$ over the time intervals $0 \leq t \leq 5$ and $0 \leq t \leq 8$, respectively, using different orders of truncation $M$. Observe that both values of $\alpha \in] 0,1]$, hence they correspond to Case I. From both graphical representations we observe that the approximations of the mean and the standard deviation converge over the whole interval. Moreover, these approximations improve as $M$ increases.

In Tables 1 and 2 we have collected the figures of relative errors of the approximations of the mean and standard deviation defined in (42) and (43), respectively. Both tables correspond to $\alpha=0.3$. We observe that for $t$ fixed both errors decrease as $M$ increases, while for a fixed truncation order $M$ these errors increase as $t$ departs from the origin $t=0$. An analogous analysis corresponding to $\alpha=0.7$ is shown in Tables 3 and 4 . In these tables, the numerical results are only shown in several points placed near the right-end of the interval $0 \leq t \leq 8$ in order to better observe how evolves that error and to account for its maximum value. Specifically, we have listed the relative errors for $t=4,5,6,7,8$, just to be clearer.

Example 6. This example illustrates Case I, corresponding to $\alpha \in] 0,1]$, when diffusion coefficient $\lambda$ is an unbounded random variable. Let us consider the random fractional IVP (1), where 
Figure 1: Approximations of the mean (left) and the standard deviation (right) of the solution stochastic process to the random fractional IVP (1) with $\alpha=0.3$ (Case I) taking different orders of truncation $M$ over the time interval [0,5] in the context of Example 5.

\begin{tabular}{|c|c|c|c|c|c|}
\hline RE (Mean) $(t ; M)$ & $t=1$ & $t=2$ & $t=3$ & $t=4$ & $t=5$ \\
\hline$M=15$ & $4.079521 \mathrm{e}-05$ & $6.947839 \mathrm{e}-04$ & $3.297477 \mathrm{e}-03$ & $9.189631 \mathrm{e}-03$ & $1.905735 \mathrm{e}-02$ \\
\hline$M=20$ & $1.419749 \mathrm{e}-06$ & $6.827955 \mathrm{e}-05$ & $5.907660 \mathrm{e}-04$ & $2.484655 \mathrm{e}-03$ & $6.938988 \mathrm{e}-03$ \\
\hline$M=25$ & $4.034508 \mathrm{e}-08$ & $5.487145 \mathrm{e}-06$ & $8.709276 \mathrm{e}-05$ & $5.606024 \mathrm{e}-04$ & $2.154161 \mathrm{e}-03$ \\
\hline$M=40$ & $3.261758 \mathrm{e}-13$ & $1.003650 \mathrm{e}-09$ & $9.874694 \mathrm{e}-08$ & $2.315692 \mathrm{e}-06$ & $2.412006 \mathrm{e}-05$ \\
\hline$M=50$ & $0.000000 \mathrm{e}-32$ & $1.570471 \mathrm{e}-12$ & $5.214614 \mathrm{e}-10$ & $2.898630 \mathrm{e}-08$ & $5.896433 \mathrm{e}-07$ \\
\hline
\end{tabular}

Table 1: Numerical values of the relative error (42) corresponding to the approximations of the mean of the solution stochastic process to the random IVP (1) with $\alpha=0.3$ (Case I) at different values of $t$ and $M$ in the context of Example 5 .

\begin{tabular}{|c|c|c|c|c|c|}
\hline $\mathrm{RE}(\mathrm{Sd})(t ; M)$ & $t=1$ & $t=2$ & $t=3$ & $t=4$ & $t=5$ \\
\hline$M=15$ & $9.638948 \mathrm{e}-05$ & $1.757966 \mathrm{e}-03$ & $8.611555 \mathrm{e}-03$ & $2.338490 \mathrm{e}-02$ & $4.545151 \mathrm{e}-02$ \\
\hline$M=20$ & $3.514650 \mathrm{e}-06$ & $1.914465 \mathrm{e}-04$ & $1.802353 \mathrm{e}-03$ & $7.588380 \mathrm{e}-03$ & $1.970048 \mathrm{e}-02$ \\
\hline$M=25$ & $1.032923 \mathrm{e}-07$ & $1.650448 \mathrm{e}-05$ & $2.962502 \mathrm{e}-04$ & $1.971703 \mathrm{e}-03$ & $7.119996 \mathrm{e}-03$ \\
\hline$M=40$ & $8.883801 \mathrm{e}-13$ & $3.424193 \mathrm{e}-09$ & $4.063098 \mathrm{e}-07$ & $1.050714 \mathrm{e}-05$ & $1.086227 \mathrm{e}-04$ \\
\hline$M=50$ & $0.000000 \mathrm{e}-32$ & $5.631090 \mathrm{e}-12$ & $2.312461 \mathrm{e}-09$ & $1.452893 \mathrm{e}-07$ & $3.004525 \mathrm{e}-06$ \\
\hline
\end{tabular}

Table 2: Numerical values of the relative error (43) corresponding to the standard deviation of the solution stochastic process to the random IVP (1) with $\alpha=0.3$ (Case I) at different values of $t$ and $M$ in the context of Example 5 .

Figure 2: Approximations of the mean (left) and the standard deviation (right) of the solution stochastic process to the random fractional IVP (1) with $\alpha=0.7$ (Case I) taking different orders of truncation $M$ over the time interval [0,8] in the context of Example 5.

\begin{tabular}{|c|c|c|c|c|c|}
\hline RE(Mean)(t;M) & $t=4$ & $t=5$ & $t=6$ & $t=7$ & $t=8$ \\
\hline$M=11$ & $2.834329 \mathrm{e}-03$ & $9.606890 \mathrm{e}-03$ & $2.328737 \mathrm{e}-02$ & $4.504716 \mathrm{e}-02$ & $7.455311 \mathrm{e}-02$ \\
\hline$M=12$ & $1.349031 \mathrm{e}-03$ & $5.309681 \mathrm{e}-03$ & $1.442739 \mathrm{e}-02$ & $3.044101 \mathrm{e}-02$ & $5.379721 \mathrm{e}-02$ \\
\hline$M=13$ & $6.168547 \mathrm{e}-04$ & $2.827171 \mathrm{e}-03$ & $8.649223 \mathrm{e}-03$ & $2.001286 \mathrm{e}-02$ & $3.797259 \mathrm{e}-02$ \\
\hline$M=14$ & $2.714154 \mathrm{e}-04$ & $1.451050 \mathrm{e}-03$ & $5.014414 \mathrm{e}-03$ & $1.278055 \mathrm{e}-02$ & $2.616524 \mathrm{e}-02$ \\
\hline$M=20$ & $9.626787 \mathrm{e}-07$ & $1.310833 \mathrm{e}-05$ & $9.664635 \mathrm{e}-05$ & $4.614136 \mathrm{e}-04$ & $1.592402 \mathrm{e}-03$ \\
\hline
\end{tabular}

Table 3: Numerical values of the relative error (42) corresponding to the mean of the solution stochastic process to the random IVP (1) with $\alpha=0.7$ (Case I) at different values of $t$ and $M$ in the context of Example 5.

\begin{tabular}{|c|c|c|c|c|c|}
\hline $\mathrm{RE}(\mathrm{Sd})(t ; M)$ & $t=4$ & $t=5$ & $t=6$ & $t=7$ & $t=8$ \\
\hline$M=11$ & $4.974931 \mathrm{e}-03$ & $1.682744 \mathrm{e}-02$ & $3.957739 \mathrm{e}-02$ & $7.319679 \mathrm{e}-02$ & $1.155156 \mathrm{e}-01$ \\
\hline$M=12$ & $2.444194 \mathrm{e}-03$ & $9.685880 \mathrm{e}-03$ & $2.561774 \mathrm{e}-02$ & $5.156699 \mathrm{e}-02$ & $8.645137 \mathrm{e}-02$ \\
\hline$M=13$ & $1.149708 \mathrm{e}-03$ & $5.353075 \mathrm{e}-03$ & $1.601557 \mathrm{e}-02$ & $3.533860 \mathrm{e}-02$ & $6.335321 \mathrm{e}-02$ \\
\hline$M=14$ & $5.188710 \mathrm{e}-04$ & $2.842382 \mathrm{e}-03$ & $9.660322 \mathrm{e}-03$ & $2.350868 \mathrm{e}-02$ & $4.535487 \mathrm{e}-02$ \\
\hline$M=20$ & $2.058083 \mathrm{e}-06$ & $2.984451 \mathrm{e}-05$ & $2.239663 \mathrm{e}-04$ & $1.045773 \mathrm{e}-03$ & $3.432793 \mathrm{e}-03$ \\
\hline
\end{tabular}

Table 4: Numerical values of the relative error (43) corresponding to the standard deviation of the solution stochastic process to the random IVP (1) with $\alpha=0.7$ (Case I) at different values of $t$ and $M$ in the context of Example 5. 


\begin{tabular}{|c|c|c|c|c|c|}
\hline $\operatorname{RE}(\operatorname{mean})(t ; M)$ & $t=6$ & $t=7$ & $t=8$ & $t=9$ & $t=10$ \\
\hline$M=15$ & $1.787302 \mathrm{e}-06$ & $6.526650 \mathrm{e}-06$ & $1.986069 \mathrm{e}-05$ & $5.253359 \mathrm{e}-05$ & $1.242889 \mathrm{e}-04$ \\
\hline$M=20$ & $3.228105 \mathrm{e}-08$ & $1.871873 \mathrm{e}-07$ & $8.502444 \mathrm{e}-07$ & $3.201927 \mathrm{e}-06$ & $1.038967 \mathrm{e}-05$ \\
\hline$M=25$ & $3.122633 \mathrm{e}-10$ & $2.875344 \mathrm{e}-09$ & $1.949542 \mathrm{e}-08$ & $1.045336 \mathrm{e}-07$ & $4.652778 \mathrm{e}-07$ \\
\hline$M=40$ & $8.693692 \mathrm{e}-16$ & $3.250538 \mathrm{e}-14$ & $7.327634 \mathrm{e}-13$ & $1.134049 \mathrm{e}-11$ & $1.302890 \mathrm{e}-10$ \\
\hline$M=50$ & $0.000000 \mathrm{e}-32$ & $0.000000 \mathrm{e}-32$ & $3.626644 \mathrm{e}-16$ & $1.478768 \mathrm{e}-14$ & $3.211297 \mathrm{e}-13$ \\
\hline
\end{tabular}

Table 5: Numerical values of the relative error (42) corresponding to the mean of the stochastic process to the random IVP (1) with $\alpha=0.6$ (Case I) in different values of $t$ and $M$ in the context of Example 6.

\begin{tabular}{|c|c|c|c|c|c|}
\hline $\mathrm{RE}(\mathrm{Sd})(t ; M)$ & $t=6$ & $t=7$ & $t=8$ & $t=9$ & $t=10$ \\
\hline$M=15$ & $5.637725 \mathrm{e}-05$ & $2.633044 \mathrm{e}-04$ & $9.870608 \mathrm{e}-04$ & $3.057817 \mathrm{e}-03$ & $7.947836 \mathrm{e}-03$ \\
\hline$M=20$ & $1.702671 \mathrm{e}-06$ & $1.447403 \mathrm{e}-05$ & $9.378429 \mathrm{e}-05$ & $4.811486 \mathrm{e}-04$ & $1.975614 \mathrm{e}-03$ \\
\hline$M=25$ & $4.642409 \mathrm{e}-08$ & $7.069181 \mathrm{e}-07$ & $7.818432 \mathrm{e}-06$ & $6.610486 \mathrm{e}-05$ & $4.333418 \mathrm{e}-04$ \\
\hline$M=40$ & $5.354437 \mathrm{e}-13$ & $4.653621 \mathrm{e}-11$ & $2.484909 \mathrm{e}-09$ & $9.011051 \mathrm{e}-08$ & $2.327453 \mathrm{e}-06$ \\
\hline$M=50$ & $0.000000 \mathrm{e}-32$ & $5.305710 \mathrm{e}-14$ & $8.013567 \mathrm{e}-12$ & $7.473559 \mathrm{e}-10$ & $4.663530 \mathrm{e}-08$ \\
\hline
\end{tabular}

Table 6: Numerical values of the relative error (43) corresponding to the standard deviation of the stochastic process to the random IVP (1) with $\alpha=0.6$ (Case I) in different values of $t$ and $M$ in the context of Example 6.

- $\beta_{0}$ is a Gamma random variable of mean $1 / 5$ and variance $1 / 25$, i.e. $\beta_{0} \sim G a(1 ; 1 / 5)$;

- $\gamma$ is a Beta random variable of mean $1 / 4$ and variance $1 / 50, \lambda \sim B e(67 / 32 ; 201 / 32)$ and

- $\lambda$ is a Gaussian random variable with zero mean and standard deviation $1 / 10, \gamma \sim$ $N\left(0 ;(1 / 10)^{2}\right)$.

We will also assume that $\beta_{0}, \gamma$ and $\lambda$ are independent random variables. Observe that in this example $\lambda$ is an unbounded random variable and, according to Example 2, it satisfies hypothesis $\hat{\mathbf{H}} 2$ with $p=1 / 2, H=\sqrt{2} / 10$ and $\eta=1 / 10$. Hypothesis $\mathbf{H 1}$ also fulfils because all input data are assumed to be independent and they have finite variance. Therefore, according to Th. 1 , the random generalized power series solution $Y(t)$, given by (17), is mean square convergent in a domain that depends on the relationship between $p=1 / 2$ and $\alpha$. In this example, we will only consider the Case I, thus $\alpha \in] 0,1]$. Specifically, for $\alpha \in] 1 / 2,1]$, that is, when $p<\alpha, Y(t)$ is mean square convergent for all $t \geq 0$, and, as a consequence, the approximations (46) and (47) for the mean and the variance (or standard deviation), respectively, will also converge for all $t \geq 0$. While if $\alpha=p=1 / 2, Y(t)$ is mean square convergent over the domain $0 \leq t<25$. Notice that the right-end value of this interval corresponds to $\alpha / H^{1 / \alpha}$. In this case, it is guaranteed that the approximations of both the mean and the variance will converge, at least, in this same interval $0 \leq t<25$, although this domain could be larger. This question will be further discussed later.

Firstly, we illustrate the former finding in Fig. 3 where we have taken $\alpha=0.6$ (Case I) as the fractional order of the derivative. In this graphical representation, we have plotted approximations of the mean and the standard deviation over the time interval $0 \leq t \leq 30$ using different orders of truncation M. In Tables 5 and 6 the numerical values of relative errors, defined in (42) and (43), at some selected values are shown. From these figures we can conclude the proposed method gives good and reliable approximations.

Secondly, we show and analyze the results obtained in the case that $p=\alpha=1 / 2$. On the leftside of Fig. 4 we have plotted the approximations of the mean over the time interval $0 \leq t \leq 60$ for 
Figure 3: Approximations of the mean (left) and the standard deviation (right) of the solution stochastic process to the random fractional IVP (1) with $\alpha=0.6$ (Case I) taking different orders of truncation $M$ over the time interval [0,30] in the context of Example 6.

Figure 4: Approximations of the mean (left) and the standard deviation (right) of the solution stochastic process to the random fractional IVP (1) with $\alpha=0.5$ (Case I) using different orders of truncation $M$ over the time intervals $[0,60]$ and $[0,30]$, respectively in the context of Example 6 . over a shorter interval, namely $0 \leq t \leq 30$ (see right-side of Fig. 4). This is an important point in our analysis regarding the case where mean square convergence takes place in a bounded interval, i.e. when $p=\alpha=1 / 2$ (see Th. 1). Observe that, according to this theorem, the approximations of the mean and the variance (standard deviation) of the solution have the same domain of convergence. This domain is inferred from the one where mean square convergence takes place. If we revise the proof of Th. 1 , we can realize that it provides a sufficient condition for mean square convergence which relies upon the construction of a convergent majorizing series. Although the result is fair general, it does not guarantee the domain of mean square convergence of the solution stochastic process $Y(t)$ (and hence of the approximations of its mean and variance), could be larger. In order to illustrate this issue, now we will show, with input we have computed and plotted the relatives errors (42) and (43) of approximations for the mean and the standard deviation. The graphical representation of these errors are shown in Figure 5 using different orders of truncation $M=50,60,70,80,90$. For the sake of clarity in this plot we have included a zoom of around the critical points $t=50$ (for the mean) and $t=25$ (for the standard deviation). From this plot, we clearly observe that divergence of approximations of the mean and the standard deviation occur after the critical points $t=50$ and $t=25$, respectively.

Figure 5: Relative errors, given in (42) and (43), of the approximations of the mean (left) and the standard deviation (right) of the solution stochastic process to the random fractional IVP (1) with $\alpha=0.5$ (Case I) using different orders of truncation $M$ over the time intervals $[0,60]$ and $[0,30]$, respectively, in the context of Example 6 . For the sake of clarity, in both plots, we present a zoom around of the end-points $t=50$ and $t=25$ of the convergence regions for the approximations of the mean and standard deviation, respectively.

different values of $M$, while the approximations of the standard deviation have been represented

As expected, the interval of convergence of the standard deviation matches the one inferred from the analysis of the mean square convergence. While the interval of convergence to the mean is larger. Now, we justify this latter numerical result using analytic arguments. This fact is intuitive since mean square convergence involves information of the second order moment (which is related to the variance/standard deviation) rather than first order moment (related to the mean). To completely support this intuition, we now prove that the interval of convergence of the deterministic series that provides approximations for the mean, given by (46) with $M \rightarrow+\infty$, is exactly $0 \leq t<50$. To this end, its sufficient to study the first series defined in (46), since the analysis of the second series is similar. Taking into account expression (9) for the statistical moments of random variable $\lambda$, it is clear that series is made up only of non-negative terms for 
all $m \geq 0$, and it has the following form

$$
\sum_{m \geq 0} \hat{\delta}_{m}(t), \quad \hat{\delta}_{m}(t):=\frac{\mathbb{E}\left[\lambda^{2 m}\right]}{\Gamma(2 \alpha m+1)} t^{2 \alpha m},
$$

Using the Stirling's approximation (15) and applying the ratio test, observe that

$$
\begin{aligned}
\lim _{m \rightarrow+\infty} \frac{\hat{\delta}_{m+1}(t)}{\hat{\delta}_{m}(t)} & =\lim _{m \rightarrow+\infty} \frac{(2 m+2)(2 m+1) \sigma^{2}}{2(m+1)} \frac{\Gamma(2 \alpha m+1)}{\Gamma(2 \alpha(m+1)+1)} t^{2 \alpha} \\
& =\frac{\sigma^{2} t^{2 \alpha}}{2} \lim _{m \rightarrow+\infty}\left(\frac{(2 m+2)(2 m+1)}{m+1}\right)\left(\lim _{m \rightarrow+\infty} \frac{\Gamma(2 \alpha m+1)}{\Gamma(2 \alpha(m+1)+1)}\right) \\
& =\frac{\sigma^{2} t^{2 \alpha}}{2} \lim _{m \rightarrow+\infty}\left(\frac{(2 m+2)(2 m+1)}{m+1}\right)\left(\lim _{m \rightarrow+\infty} \frac{(2 \alpha m)^{2 \alpha m} \mathrm{e}^{-2 \alpha m} \sqrt{2 \pi(2 \alpha m)}}{(2 \alpha(m+1))^{2 \alpha(m+1)} \mathrm{e}^{-2 \alpha(m+1)} \sqrt{2 \pi(2 \alpha(m+1))}}\right) \\
& =\frac{\sigma^{2} t^{2 \alpha}}{2} \lim _{m \rightarrow+\infty}\left(\frac{(2 m+2)(2 m+1)}{m+1}\right)\left(\lim _{m \rightarrow+\infty} \frac{1}{(2 \alpha(m+1))^{2 \alpha}}\right) \\
& =\frac{\sigma^{2} t^{2 \alpha}}{2(2 \alpha)^{2 \alpha}} \lim _{m \rightarrow+\infty}\left(\frac{(2 m+2)(2 m+1)}{(m+1)^{2 \alpha+1}}\right) \\
& =\left\{\begin{array}{cl}
0 & \text { if } \quad \alpha>1 / 2, \\
2 \sigma^{2} t & \text { if } \quad \alpha<1 / 2,
\end{array}\right.
\end{aligned}
$$

Therefore, according to the ratio test, if $\alpha<1 / 2$ the domain of convergence of the first series of (46) (and hence of the full series (46)) is $t>0$; if $\alpha>1 / 2$ there is no convergence for all $t>0$, and if $\alpha=1 / 2$ the domain of convergence is $0<t<1 /\left(2 \sigma^{2}\right)$. Thus, in this latter case if $\sigma=1 / 10$, such as it has been chosen in our numerical experiments, the domain of convergence of the series (46) (with $M \rightarrow+\infty$ ), defining the approximations of the mean is $0 \leq t<50$. This fully agrees with the results shown in Fig. 4 and Fig. 5.

Example 7. This example illustrates Case II, corresponding to $\alpha=1.2 \in] 1,2]$, when diffusion coefficient $\lambda$ is an unbounded random variable. Let us consider the random fractional IVP (1), where

- $\beta_{0}$ and $\beta_{1}$ are Gamma random variables of mean $1 / 2$ and variance $1 / 2$, i.e. $\beta_{0} \sim G a(1 / 2 ; 1)$;

- $\gamma$ is a Gaussian random variable of mean $1 / 2$ and variance $1 / 2, \lambda \sim N\left(1 / 2 ;(\sqrt{2} / 2)^{2}\right)$ and

- $\lambda$ is an Exponential random variable with mean $1 / 6$ and variance $1 / 36, \lambda \sim \operatorname{Exp}(6)$.

We assume that all input data $\beta_{0}, \beta_{1}, \gamma$ and $\lambda$ are mutually independent random variables. Hence, hypothesis $\hat{\mathbf{H} 1}$ is fulfilled. Since $\lambda$ is an Exponential random variable, it is an unbounded and, by Example 3, it satisfies hypothesis $\hat{\mathbf{H}} 2$ with $p=1, H=1 / 3$ and $\eta=\sqrt{2} / 6$. Therefore, as $p=1<1.2=\alpha$ by Th. 2, it is known that the random generalized power series solution $Y(t)$, given by (27), is mean square convergent for all $t \geq 0$. As a consequence, the approximations for that for the mean and the variance (or standard deviation), given by (31)-(41), respectively, will converge for all $t \geq 0$. Approximations for these statistical moments are shown in Fig. 6 using the following orders of truncation $M=5,7,10,12,15$. We observe the convergence over 
the whole intervals. In Fig. 7, we show an approximation to the correlation coefficient function associated to the solution stochastic process. This surface has been built taking $M=20$ in the following expression

$$
\rho_{Y_{M}}(t, s)=\frac{\mathbb{C}_{Y_{M}, Y_{M}}(t, s)}{\sqrt{\mathbb{V}\left[Y_{M}(t)\right]} \times \sqrt{\mathbb{V}\left[Y_{M}(s)\right]}} .
$$

This function measures the lineal statistical dependence between the approximations $Y_{M}(s)$ and $Y_{M}(t)$ in two different time instants $s$ and $t$. From Fig. 7, we can observe that linear statistical interdependence is stronger in points located about the diagonal $(t, t)$. For $M$ fixed, this means that random variable $Y_{M}(s)$ can be approximated by a linear function of $Y_{M}(t)$ when $s$ and $t$ are close.

Figure 6: Approximations of the mean (left) and the standard deviation (right) of the solution stochastic process to the random fractional IVP (1) with $\alpha=1.2$ (Case II) using different orders of truncation $M$ over the time intervals [0,8] and $[0,5]$, respectively in the context of Example 7.

Figure 7: Approximations of the correlation coefficient associated to the solution stochastic process to the random fractional IVP (1) with $\alpha=1.2$ (Case II) taking as order of truncation $M=20$ in the context of Example 7.

\section{Conclusions}

In this paper we have extended some results recently obtained for the random linear fractional differential equation using the mean square calculus and the random Caputo derivative. We have constructed a solution stochastic process for that class of equations by means of a random generalized power series. Furthermore, we have given mild conditions in order to guarantee its mean square convergence. Afterwards, we have provided closed-form expressions for approximations of its main statistical functions (mean, variance, covariance and cross-covariance). The analysis permits to enlarge the family of random variables playing the role of diffusion coefficient for that class of fractional differential equation. Specifically, significant unbounded random variables such as Gaussian and Exponential are included in our hypotheses. We think that many of the ideas developed throughout our analysis can be used in future research to deal with other class of random fractional differential equations.

\section{Acknowledgements}

This work has been partially supported by the Ministerio de Economía, Industria y Competitividad grant MTM2017-89664-P.

\section{Conflict of Interest Statement}

The authors declare that there is no conflict of interests regarding the publication of this article. 


$$
\begin{aligned}
\mathbb{V}\left[Y_{M}(t)\right]= & \mathbb{E}\left[\left(\beta_{0}\right)^{2}\right] \sum_{m=0}^{M} \sum_{n=0}^{M} \frac{\mathbb{E}\left[\lambda^{m+n}\right]}{\Gamma(\alpha m+1) \Gamma(\alpha n+1)} t^{\alpha(m+n)} \\
& -\left(\mathbb{E}\left[\beta_{0}\right]\right)^{2}\left(\sum_{m=0}^{M} \frac{\mathbb{E}\left[\lambda^{m}\right]}{\Gamma(\alpha m+1)} t^{\alpha m}\right)\left(\sum_{n=0}^{M} \frac{\mathbb{E}\left[\lambda^{n}\right]}{\Gamma(\alpha n+1)} t^{\alpha n}\right) \\
& +\mathbb{E}\left[\beta_{0}\right] \mathbb{E}[\gamma] \sum_{m=0}^{M} \sum_{n=1}^{M} \frac{\left(\mathbb{E}\left[\lambda^{m+n-1}\right]\right)}{\Gamma(\alpha m+1) \Gamma(\alpha n+1)} t^{\alpha(m+n)} \\
& -\mathbb{E}\left[\beta_{0}\right] \mathbb{E}[\gamma]\left(\sum_{m=0}^{M} \frac{\mathbb{E}\left[\lambda^{m}\right]}{\Gamma(\alpha m+1)} t^{\alpha m}\right)\left(\sum_{n=1}^{M} \frac{\mathbb{E}\left[\lambda^{n-1}\right]}{\Gamma(\alpha n+1)} t^{\alpha n}\right) \\
& +\mathbb{E}\left[\beta_{0}\right] \mathbb{E}[\gamma] \sum_{m=1}^{M} \sum_{n=0}^{M} \frac{\mathbb{E}\left[\lambda^{m+n-1}\right]}{\Gamma(\alpha m+1) \Gamma(\alpha n+1)} t^{\alpha(m+n)} \\
& -\mathbb{E}\left[\beta_{0}\right] \mathbb{E}[\gamma]\left(\sum_{m=1}^{M} \frac{\mathbb{E}\left[\lambda^{m-1}\right]}{\Gamma(\alpha m+1)} t^{\alpha m}\right)\left(\sum_{n=0}^{M} \frac{\mathbb{E}\left[\lambda^{n}\right]}{\Gamma(\alpha n+1)} t^{\alpha n}\right) \\
& +\mathbb{E}\left[\gamma^{2}\right] \sum_{m=1}^{M} \sum_{n=1}^{M} \frac{\mathbb{E}\left[\lambda^{m+n-2}\right]}{\Gamma(\alpha m+1) \Gamma(\alpha n+1)} t^{\alpha(m+n)} \\
& -(\mathbb{E}[\gamma])^{2}\left(\sum_{m=1}^{M} \frac{\mathbb{E}\left[\lambda^{m-1}\right]}{\Gamma(\alpha m+1)} t^{\alpha m}\right)\left(\sum_{n=1}^{M} \frac{\mathbb{E}\left[\lambda^{n-1}\right]}{\Gamma(\alpha n+1)} t^{\alpha n}\right) .
\end{aligned}
$$

\section{References}

[1] B. Øksendal, Stochastic Differential Equations: An Introduction with Applications, Springer-Verlag, BerlinHeidelberg, 2003.

[2] P. Kloeden, E. Platen, Numerical Solution of Stochastic Differential Equations, Applications of Mathematics, Springer, Berlin, 1992.

[3] T. T. Soong, Random Differential Equations in Science and Engineering, Academic Press, New York, 1973.

[4] L. Villafuerte, C. A. Braumann, J. C. Cortés, L. Jódar, Random differential operational calculus: Theory and applications, Computers \& Mathematics with Applications 59 (1) (2010) 115-125.

[5] T. Neckel, F. Rupp, Random Differential Equations in Scientific Computing, Versita, London, 2013.

[6] K. Nouri, H. Ranjbar, Mean square convergence of the numerical solution of random differential equations, Mediterranean Journal of Mathematics (2014) 1-18. doi:10.1007/s00009-014-0452-8. 
[7] L. T. Santos, F. A. Dorini, M. C. C. Cunha, The probability density function to the random linear transport equation, Applied Mathematics and Computation 216 (5) (2010) 1524-1530. doi:10.16/j.amc.2010.03.001.

[8] A. A. Kilbas, H. M. Srivastava, J. J. Trujillo, Theory and Applications of Fractional Differential Equations, Elsevier Science, The Netherlands, 2006.

[9] Y. Khan, Q. Wu, N. Faraz, A. Yildirim, M. Madani, New fractional analytical approach via a modified RiemannLiouville derivative, Applied Mathematics Letters 25 (2012) 1729-1733. doi:10.1016/j.aml.2012.02.001.

[10] Y. Khan, M. Fardi, K. Sayevand, M. Ghasemi, Solution of nonlinear fractional differential equations using an efficient approach, Neural Computing and Applications 24 (2014) 187-192. doi:10.1007/s00521-012-1208-7.

[11] A. Debbouche, J. Nieto, Sobolev type fractional abstract evolution equations with nonlocal conditions and optimal multi-controls, Applied Mathematics and Computation 245 (2014) 74-85. doi:10.1016/j.amc.2014.07.073.

[12] K. Mourad, A. Debbouche, Complete controllability of nonlocal fractional stochastic differential evolution equations with Poisson jumps in Hilbert spaces, International Journal of Advances in Applied Mathematics and Mechanics 3 (1) (2015) 41-48.

[13] G. González-Parra, B. M. Chen-Charpentier, A. J. Arenas, Polynomial chaos for random fractional order differential equations, Applied Mathematics and Computation 226 (2014) 123-130. doi:10.1016/j.amc.2013.10.051.

[14] A. K. Golmankhaneh, N. A. Porghoveh, D. Baleanu, Mean square solutions of second-order random differential equations by using homotopy analysis method, Romanian Reports in Physics 65 (2013) 350-362.

[15] A. K. Golmankhaneh, R. Arefi, D. Baleanu, Synchronization in a nonidentical fractional order of a proposed modified system, Journal of Vibration and Control 21 (2015) 1154-1161.

[16] V. Lupulescu, D. O'Reagan, G. u. Rahman, Existence results for random fractional differential equations, Opuscula Mathematica 34 (4) (2014) 813-825. doi:10.7494/OpMath.2014.34.4.813.

[17] V. Lupulescu, K. N. Ntouyas, Random fractional differential equations, International Electronic Journal of Pure and Applied Mathematics 4 (2) (2012) 119-136.

[18] L. Acedo, C. Burgos, J.-C. Cortés, R. Villanueva, Probabilistic prediction of outbreaks of meningococcus W-135 infections over the next few years in Spain, Physica A: Statistical Mechanics and its Applications 486 (2017) 106-117. doi:10.1016/j.physa.2017.05.043.

19] C. Burgos, J. C. Cortés, L. Villafuerte, R. J. Villanueva, Extending the deterministic Riemann-Liouville and Caputo operators to the random framework: A mean square approach with applications to solve random fractional differential equations, Chaos, Solitons \& Fractalsdoi:10.1016/j.chaos.2017.02.008.

[20] M. Loève, Probability Theory I, Vol. 45 of Graduate Texts in Mathematics, Springer-Verlag, New York, 1977.

[21] A. Papoulis, S. U. Pillai, Probability, Random Variables and Stochastic Processes, 4th Edition, McGraw-Hill, New York, 2002.

[22] G. R. Grimmett, D. R. Stirzaker, Probability and Random Processes, Clarendon Press, New York, 2000.

[23] J.-C. Cortés, L. Villafuerte, C. Burgos, A mean square chain rule and its applications in solving the random Chebyschev differential equation, Mediterranean Journal of Mathematics 13 (6) (2017) 3817-3836. doi:10.1007/s00009-017-0853-6. 